\title{
Magnetic knots of deconfined CP-odd matter in heavy-ion collisions
}

\author{
M. N. Chernodub* \\ CNRS, Laboratoire de Mathématiques et Physique Théorique, Université François-Rabelais, \\ Fédération Denis Poisson - CNRS, Parc de Grandmont, Université de Tours, 37200 France \\ Department of Physics and Astronomy, University of Gent, Krijgslaan 281, S9, B-9000 Gent, \\ Belgium \\ E-mail: maxim. chernodubelmpt.univ-tours.fr
}

\begin{abstract}
We point out that metastable knots of deconfined hot quark matter may exist in the quark-gluon plasma due to local parity violating effects. These knots are stabilized by strong magnetic fields which induce electric currents via the chiral magnetic effect. The magnetic field in the knot resembles the spheromak plasma state which appears in the nuclear fusion devices. The size of the knot is inversely proportional to the chiral conductivity of the plasma, and the parity (P) and charge-parity (CP) symmetries are broken inside the knot. We argue that these knots may be created in noncentral heavy-ion collisions.
\end{abstract}

35th International Conference of High Energy Physics - ICHEP2010,

July 22-28, 2010

Paris France

*This work was partially supported by the grant ANR-10-JCJC-0408 HYPERMAG. 
Noncentral heavy-ion collisions may generate very strong magnetic field with the strength of the order of the hadronic scale. In LHC Pb-Pb collisions the strength of the magnetic field may reach the value $e B_{\mathrm{LHC}}^{\max } \gtrsim 15 m_{\pi}^{2}$, where $m_{\pi} \approx 140 \mathrm{MeV}$ is the pion mass [1]. A magnetic field of such strength should affect dynamics of the charged constituents of the quark-gluon plasma and consequently lead to several observable effects in heavy-ion collisions. For example, the quarkgluon plasma may contain parity-violating domains due to instanton and sphaleron transitions. In the parity-violating background the external magnetic field $B$ leads to generation of a quark's electric current $\vec{j}$ via the so called chiral magnetic effect (CME) [2]:

$$
\vec{j}=\sigma_{\chi} \vec{B},
$$

where $\sigma_{\chi}$ is the chiral electric conductivity which depends on properties of the quark-gluon plasma (the external electric field is assumed to be absent, $\vec{E}=0$ ). The first evidence of the existence of this exotic effect was recently reported by the STAR collaboration at the RHIC facility [3].

The transport law (1) leads to existence of certain nontrivial metastable states. A combination of Eq. (1) with the classical Maxwell equations provides us with the well known system of equations which plays an important role in usual (electromagnetic) plasma physics. Solutions of this system are usually called Chandrasekhar-Kendall states in astrophysical plasmas [4]. In the theory of nuclear fusion devices, the solutions are known as Taylor ("spheromak") states [5], which are the "natural-end configurations" in the plasma equilibrium. We suggest that due to the parity violating effects similar states may arise in the quark-gluon plasma created in heavy-ion collisions [6].

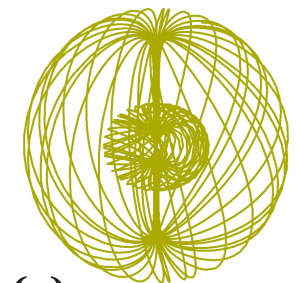

(a)

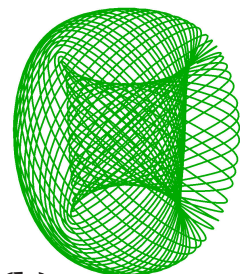

(b)

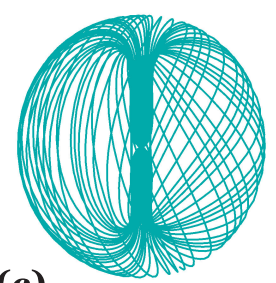

(c)

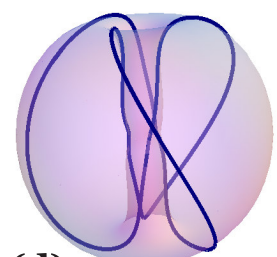

(d)

Figure 1: Typical structures formed by the lines of the magnetic field (the illustration is from Ref. [6]).

The spheromak state is a complicated compact structure of selflinked (knotted) magnetic lines. The simplest state (we call it as "knot") is a spherial solution of the finite radius $R \sim 1 / \sigma_{\chi}$. Typical lines of the magnetic field in these knots are illustrated in Figure 1. In heavy-ion collisions the knots may probably be formed in the parity-broken domains. Further details can be found in Ref. [6].

\section{References}

[1] V. Skokov, A. Y. Illarionov and V. Toneev, Int. J. Mod. Phys. A 24, 5925 (2009) [arXiv:0907.1396].

[2] K. Fukushima, D.E. Kharzeev, and H.J. Warringa, Phys. Rev. D 78, 074033 (2008) [arXiv:0808.3382].

[3] B. I. Abelev et al. [STAR Collaboration], Phys. Rev. Lett. 103, 251601 (2009) [arXiv:0909.1739].

[4] S. Chandrasekhar and P.C. Kendall, Astrophys. J. 126, 457 (1957).

[5] J.B. Taylor, Phys. Rev. Lett. 33, 1139 (1974).

[6] M. N. Chernodub, arXiv:1002.1473 [nucl-th]. 